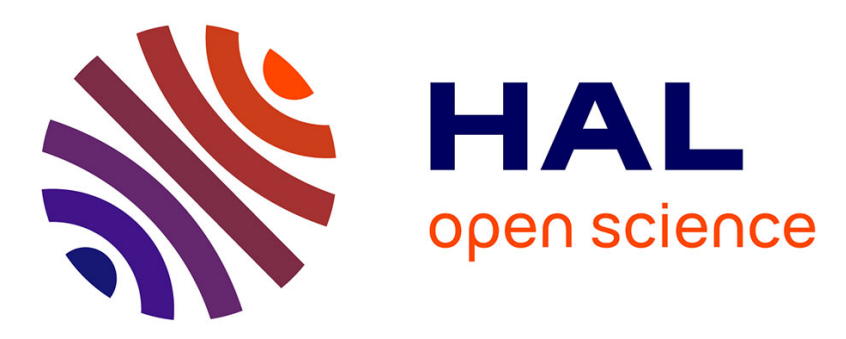

\title{
From steady-state to cyclic metal forming processes
}

Pierre Montmitonnet

\section{To cite this version:}

Pierre Montmitonnet. From steady-state to cyclic metal forming processes. Materials Processing and Design, Modeling, Simulation and Applications, NUMIFORM '07: 9th International Conference on Numerical Methods in Industrial Forming Processes, Jun 2007, Porto, Portugal. p. 209-214, 10.1063/1.2740813 . hal-00510558

\section{HAL Id: hal-00510558 \\ https://hal-mines-paristech.archives-ouvertes.fr/hal-00510558}

Submitted on 1 Apr 2011

HAL is a multi-disciplinary open access archive for the deposit and dissemination of scientific research documents, whether they are published or not. The documents may come from teaching and research institutions in France or abroad, or from public or private research centers.
L'archive ouverte pluridisciplinaire HAL, est destinée au dépôt et à la diffusion de documents scientifiques de niveau recherche, publiés ou non, émanant des établissements d'enseignement et de recherche français ou étrangers, des laboratoires publics ou privés. 


\title{
From Steady-State To Cyclic Metal Forming Processes
}

\author{
Pierre Montmitonnet \\ Cemef, UMR Ecole des Mines de Paris / CNRS n ${ }^{\circ} 7635-$ \\ Ecole des Mines, BP 207, 06904 Sophia-Antipolis CEDEX, France
}

\begin{abstract}
Continuous processes often exhibit a high proportion of steady state, and have been modeled with steadystate formulations for thirty years, resulting in very CPU-time efficient computations. On the other hand, incremental forming processes generally remain a challenge for FEM software, because of the local nature of deformation compared with the size of the part to be formed, and of the large number of deformation steps needed. Among them however, certain semi-continuous metal forming processes can be characterized as periodic, or cyclic. In this case, an efficient computational strategy can be derived from the ideas behind the steady-state models. This will be illustrated with the example of pilgering, a seamless tube cold rolling process.
\end{abstract}

Keywords: cyclic processes; FEM modelling ; material history.

\section{INTRODUCTION}

Incremental processes, in which deformation is split in a series of steps (roll forming, ring rolling, cross wedge rolling, spinning, rotary forging...) remain a challenge even for advanced numerical techniques [1]. This is due to the local character of deformation, which requires fine local meshing in a generally large system, and to the large number of steps needed to form the whole part. Moreover, each step is a time-dependent thermo-mechanical process, requiring a time-stepping numerical scheme.

In some cases, a cyclic character emerges from the analysis of the process. This is the case for the cold pilgering of tubes $[2,3]$. This means that if the formulation if properly coined, it may prove sufficient to compute only one or a few of the numerous cycles in which the whole process consists. In this respect, such processes are intermediate between fully incremental forming and processes presenting a dominant steady state (strip / bar rolling, extrusion...). In the following, we describe an intermediate computational strategy, recycling part of the ideas behind the numerous steady state models proposed for rolling (e.g. [4-8]), adapted to the cyclic situation, resulting in a still computationally intensive approach, yet much improved in efficiency.

\section{DESCRIPTION OF PILGERING}

Pilgering is a seamless tube cold forming process. The material enters as a tubular perform with a thick wall and large diameter, and exits as a thinner and smaller tube: both thickness and diameter are reduced, using two main kinds of tools (fig.1).

The mandrel calibrates the inner surface; it is axissymmetrical, its diameter evolves from the preform inner diameter to the pilgered tube inner diameter. Its z-profile shape is an important process parameter, controlling the progression of the radial / orthoradial strain ratio, quite important for metal integrity, microstructure and texture evolution.

The dies wear grooves, the section of which is basically a circle shifted from the symmetry plane (see fig.2). The groove section diameter evolves between the outer diameters of the preform and pilgered tube.

\section{Tool Kinematics}

The mandrel is fixed in translation as well as in rotation. The dies are rolling back and forth along the mandrel axis $\mathrm{Oz}$; their movement is controlled by a rack-and-pinion system, ensuring rolling without sliding. Thus, at a given z-position, a given groove depth always corresponds to a given mandrel diameter, leaving a well defined gap for the material. This is called "conjugation". 
Between each back-and-forth movement of the dies (a "stroke"), the preform is turned (by about $50^{\circ}$ ) and pushed forward by a few $\mathrm{mm}$. Rotation ensures that although the gap where it is rolled is not exactly axissymmetrical, the tube will exit precisely circular from the deformation zone. Furthermore, after being advanced, the preform presents at position $\mathrm{z}$ a thickness greater than the die / mandrel gap, so that the next stroke will indeed reduce and elongate the tube (fig.2). With this combination of rigid rotation and translation before the stroke, plus elongation during the stroke, it takes 50 to 100 strokes for a material point to go its helical way through the deformation zone, from the preform side to the finished tube side, being deformed sometimes in the flange area, sometimes near the groove bottom. Also note that it takes about 2500 strokes to pilger a 5.2-m long preform.

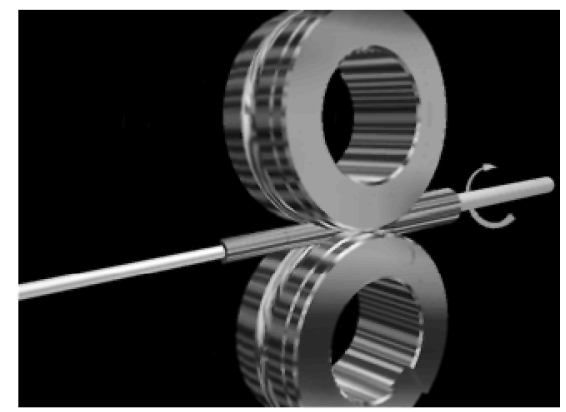

FIGURE 1. Principle of the cold pilgering process, showing the mandrel, the two dies (pictured here without their driving axes for better visibility), and the preform being pilgered (named "transition").

\section{Analysis Of The Strain And Stress Pattern}

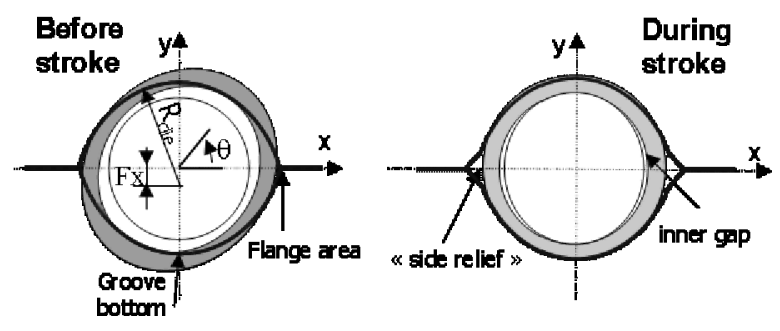

FIGURE 2. Cross-section of the tube / die / mandrel system at a given z-position. Thin line: mandrel section; thick line: die groove section; grey : section of the tube "transition".

Figure 2 compares the geometry of the system, in cross-section at a definite position $z$. Before the stroke (i.e. after turning and advancing, left), (i) the tube is thicker than the gap, (ii) its symmetry planes are turned $\approx 50^{\circ}$ with respect to those of the dies, resulting in the absence of any plane of symmetry (only central symmetry remains), (iii) the thickness is highest in the $\theta \approx 50^{\circ}$ area, which was in the flange area during the previous stroke. In the flange area, a side relief is managed to let the tube ovalize slightly without being pinched. The thickness has a minimum at $\theta \approx 140^{\circ}$, which comes from the groove bottom. As for the gap, it has a minimum at groove bottom $\left(\theta \approx 90^{\circ}\right.$ or $\left.270^{\circ}\right)$ and a maximum in the flange area $\left(\theta \approx 0\right.$ or $\left.180^{\circ}\right)$.

As a result, most of the thickness reduction occurs in a wide area around the groove bottom, with a maximum at $\theta \approx 70$ to $80^{\circ}$ (see fig.5), under a large compressive $\sigma_{\text {IT }}$. This reduction, by incompressibility, results both in orthoradial and longitudinal flow. A slight $\theta$-elongation takes place, as metal escapes the thinner gap towards the thicker areas; this flow is obviously restricted by the geometry, so that the $\theta$ stress is always strongly compressive everywhere; the metal flow is dominantly elongation in the $\mathrm{z}$-direction.

In the flange area, due to the side-relief, the available space is largest; if the preform advance is chosen small enough, the result is an absence of contact of the tube with the mandrel and die flange. Therefore, $\sigma_{\text {Ir }} \approx 0$, and the thickness increases slightly $\left(\varepsilon_{\mathrm{rr}}>0\right)$. Elongation takes place only because the material is forced to yield by the elongation of neighboring material in the groove bottom. This results in a highly tensile $\sigma_{z z}$.

\section{CYCLICITY VERSUS STEADY STATE}

Looking at fig.2 again, it is important to realize that once the tube on the right side of the figure has been turned and advanced before the next stroke, the system will be in exactly the same state as before the stroke (left part of the figure). This ensures that in spite of the semi-continuous and repetitive nature of the process, the finished product is constant and homogeneous to a good accuracy. This is true for the geometry, and also for the strains, which are just the translation of the difference between the tube and gap geometries; and therefore also for the stresses. This periodic character from a Eulerian point of view (i.e. in a referential fixed to the frame of the machine) is an essential practical and theoretical feature. It is confirmed e.g. by the vertical pilgering force recording of fig. 3 , where forward and return rolling show by distinct peaks, one big (forward) and one small (return) forming a cycle. To a good "industrial accuracy", this basic cycle repeats identically all along the rolling of a preform to the probable exception of both ends.

Of course, for the material points, there is no periodicity: strain accumulates, stresses change as the point moves helicoidally around the mandrel, sometimes in the flange area, sometimes in the groove bottom, with the different stress and strain states 
described above. So the Eulerian point of view is cyclic, the Lagrangian point of view is not. Simply, the same stress and strain patterns are cyclically reproduced, but applied to different particles.

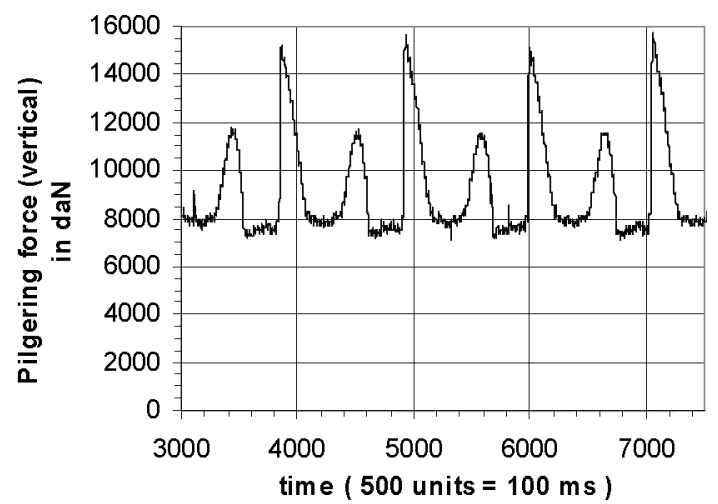

FIGURE 3. The measured pilgering force shows the periodic nature of the process. The minimum force $(8000$ $\mathrm{daN}$ ) is a pre-compression imposed to the dies to avoid the "pilgering slit" (die separation changing the gap geometry).

This is similar to what occurs in steady-state phases of continuous processes, strip rolling e.g.: the velocity, temperature, strain and stress fields are timeindependent in a fixed frame (once end effects are neglected), although material points do undergo a time-dependent history as they move along their streamlines. This analogy is the basis for the treatment we propose for such cyclic problems as pilgering.

\section{Steady-State Formulation Algorithm}

The steady-state formulations are well known. Although based on different numerical techniques, they all obey the same principle [4-8]. Focusing on the mechanics, leaving aside thermal transfer for clarity:

1) as the shape of the product during deformation is not known, choose a starting geometry $\Omega_{0}$ which will remain fixed in space (Eulerian - or quasi-Eulerian formulation). Generally the initial width is kept, but if a better guess can be made, convergence towards the final shape will be all the faster.

2) assume initial stress and strain fields to be zero (if a relevant strain map e.g. can be introduced, convergence of velocity field resolution is improved).

3) apply the boundary conditions (BCs): free surfaces, contact and friction surfaces, tensions at entry and exit.

The latter require sufficient lengths before entry and after exit to be meshed, so that the approximations on BCs do not interfere with the stress field; this is similar to Saint-Venant's principle. This sometimes (for wide strips) cumbersome condition may be relaxed using an imposed force (rather than imposed tension stress), necessarily complemented with a homogeneous velocity condition in entry/exit sections.

4) solve for the time-independent velocity $v(x, y, z)$, generally by Newton-Raphson's iterative technique.

5) from $v(x, y, z)$, compute streamlines, solving :

$$
\frac{d x}{v_{x}}=\frac{d y}{v_{y}}=\frac{d z}{v_{z}}
$$

6) integrate all history-dependent variables (strain, stress if elastic-plastic behaviour is assumed, internal or microstructural variables and functions...) along the streamlines, based on their evolution equation, e.g.:

$$
\bar{\varepsilon}=\int \dot{\bar{\varepsilon}} d t
$$

This reconstructs the material history of any particle (the Lagrangian point of view).

7) this gives a full solution, but computed on an approximate domain shape $\Omega_{0}$. An improved free surface shape can be computed, solving its stationarity equation (3) for new positions of free surface nodes in each cross-section (a variety of techniques have been proposed [4-8]):

$$
v . n=0 .
$$

A mesh regularization step may follow if spread (widening) is strong.

8) on this new domain $\Omega_{1}$, a new solution $\mathbf{v}(\mathrm{x}, \mathrm{y}, \mathrm{z})$ is sought, starting an iterative process until convergence of $\Omega$ to $\Omega_{\text {conv. }} \Omega_{\text {conv }}$ is a fixed point of the transformation, i.e. starting from $\Omega_{\text {conv }}$, computing the velocity field, solving for the shape of the free surfaces satisfying eq.(3), $\Omega_{\text {conv }}$ is resumed.

\section{Generalization to cyclic processes}

Due to the periodic nature of the pilgering process, the mechanical state (geometry, strain and stress...) at the beginning of a stroke is also a fixed point of a transformation which involves the plastic displacement field induced by the rolling of the dies and the rigid body movement of the preform before the stroke (rotation + translation). The difference is that the result required is not a time-independent state (summarized by $\mathbf{v}(\mathrm{x}, \mathrm{y}, \mathrm{z})$ ), but a whole time-dependent deformation process. The following algorithm has been derived to exploit the similarity and cope with the differences:

1) the geometry of the domain has to be initialized; the closer to reality, the faster the iterative process will converge towards the fixed point. Here, the geometry is the assumed shape of a "transition", i.e. the tube 
being formed, comprising a short section of the preform and of the finished tube. The choice has been based upon (i) the gap between the mandrel and die, zsection by z-section; (ii) where the gap is not filled (side-relief), assumptions have been derived from experimental 3D measurements of a "transition" obtained by interrupted rolling [9].

2) the strain has to be carefully initialized, as it accumulates stroke after stroke in such an incremental process. If the strain were zero, the required number of stroke computations for convergence would be the real number for a material point to cross the whole deformation zone, i.e. $\approx 70$. As will be shown, a much faster convergence can be obtained if the initial strain map is consistent with the geometry. We have chosen:

$$
\bar{\varepsilon}=\operatorname{Ln}\left(\frac{S_{0}}{S(z)}\right)
$$

where S represents the area of the cross section. This estimate (pure elongations) neglects shear components, and possible reversing strains such as thickening in the side relief / thinning in the groove bottom. The iterative process will have to correct these approximations.

3) initializing the stress to zero has been found sufficient. This is due to the behavior of residual stresses in elasto-plasticity, which decay exponentially with further imposed plastic strain [10].

4) $\mathrm{BCs}$ are to be imposed: contact / friction surfaces, free surfaces; spurious movements of the tube and preform ends are avoided, in the real process by tube guides, in the computation by canceling $\mathrm{v}_{\mathrm{x}}$ and $\mathrm{v}_{\mathrm{y}}$ components in extreme sections.

5) the streamline concept does not apply in this non-steady-state process. Particle trajectories must be computed instead. Suppose the computation of a stroke has been performed. At the last time-step, the total displacement field is stored. Take a material point at an arbitrary initial position on the preform side. Apply the abovementioned displacement, then the rigid body movement; this gives a new position in the fixed domain. Its own total displacement can be extracted by interpolation, and added as the effect of the next stroke, together with another rigid body movement; this process can be iterated until the material point leaves the deformation zone on the finished tube side. All this treatment is a post-processing of the onestroke results, requiring no further FEM computation.

6) the mechanical field history (e.g. stress $\sigma(t))$ is picked up from the result files at all successive positions determined by this post-processing along the ca. 70 (not computed) strokes, and appended to form the complete history of the material point along the ca. 70 strokes.
7) All this has been obtained by computing one stroke only. It is however important to realize that this procedure makes sense only if this computed stroke represents the real one, i.e. if the initial state is the fixed point. Due to the assumptions on initialization (of geometry, strain and stresses), this cannot be the case. This is why the one-stroke FEM computation has to be iterated a few times, until the fixed point is observed, before the abovementioned post-processing may be started. This is identical to the "free surface iterations" of steady-state formulations.

\section{APPLICATION TO PILGERING}

This procedure has been applied to the pilgering of a zircaloy 4 tube destined for the nuclear industry. The details of the operation can be found elsewhere [2, 3]. Forge $3 \circledR$ has been used as the FEM solver, and only the cyclic post-processing tool is specific. Each stroke computation comprises 1200 to 1500 time-steps and lasts about one CPU-day on a $2 \mathrm{GHz}$ PC. The first test of the procedure consists in checking that the geometry and mechanical fields do converge to a fixed point when several stroke computations are chained.

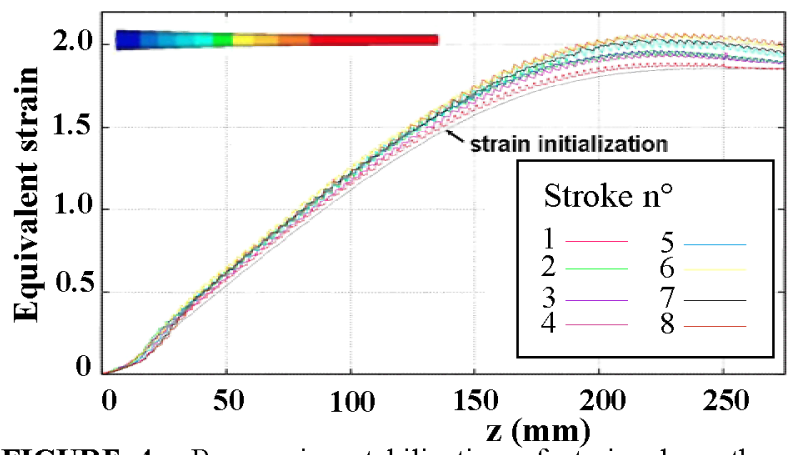

FIGURE 4. Progressive stabilization of strain along the "iterative" stroke computation process: the strain z-profile evolves from its initialization, shear-less guess to the final state. The insert represents the strain initialization, eq.(4) (blue: $\bar{\varepsilon}=0$; red: $\bar{\varepsilon}=1.8$ ).

This has been verified for all variables, but is illustrated here only for the strain, for the sake of brevity. Figure 4 shows the strain profile along a generator of the transition, the initialization strain as well as the profiles after a growing number of strokes. The strain increases in the first few strokes (up to 4 or 5), because the successive computations add the initially neglected strains; then it stabilizes.

Figure 5 presents the radial strain component on the external surface, after stroke 1 and after stroke 4. The position and intensity of the maximum strain, near the groove bottom, evolves along the iterative process, again due to the correction of initial approximations. It stabilizes after 4 strokes. In this centro-symmetrical 
process, it seems that everything in fact stabilizes when each material point has rotated by $180^{\circ}$ or more.

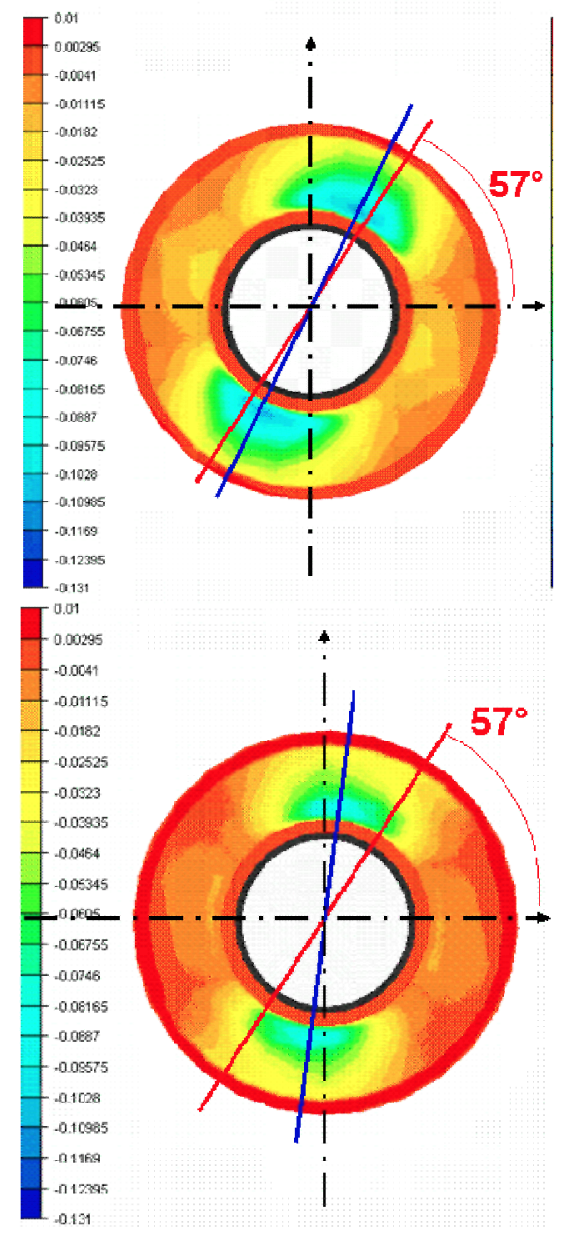

FIGURE 5. Progressive stabilization of strain along the "iterative" stroke computation process: the "transition" tube is seen from the front, showing the radial strain component on the external surface. Top: first stroke computation; bottom: $4^{\text {th }}$ stroke. The thick blue lines points to the maximum radial strain.

Once convergence is obtained, the material point mechanical history can be extracted. As an example, the $\sigma_{\mathrm{zz}}$ stress component is pictured in fig.6. It consists in an alternation of tension ( $>0$, during strokes where the point is in the side relief) and compression peaks ( $<0$, near the groove bottom). Note that each peak is in fact the whole history of one stroke, and can be finely resolved if necessary, provided every time-step or so in the (converged) one-stroke computation has been stored, in order not to degrade the information. Indeed, it has been shown [3] that storing too sparsely may decrease or erase peaks, because the stress at the very moment when the die was rolling on the material point at its position during stroke $\mathrm{n}^{\circ} n$ might be absent.

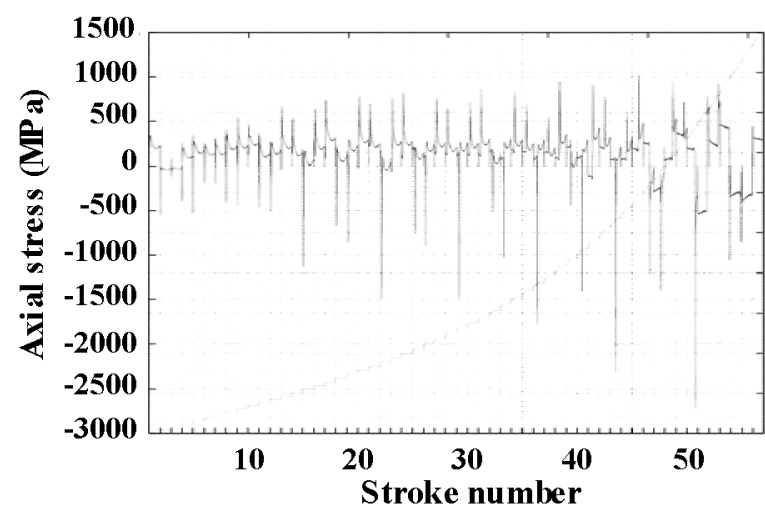

FIGURE 6. Stress history (axial $\sigma_{z z}$ component) of a material point all along its trajectory in the deformation zone (70 strokes).

These peak stresses (or peak strain components, which can be reconstructed in the same way) are essential to understand low-cycle fatigue problems encountered in pilgering, to try and design laboratory mechanical tests reproducing it, on the way to "defectfree tools".

\section{OTHER CYCLIC PROBLEMS}

There are other "cyclic" problems in the metal forming industry. Can they benefit from the same, or similar techniques? Take for instance the case of hot forging. The tool temperature is an important feature, impacting both its service life, and the heat transfer from the part to the tool. It is therefore important to account for it, but a cyclic state establishes in a few tens to a few hundred parts (fig. 7).

Computing hundreds of strokes just to be sure that the thermal regime of the die has been properly accounted for is costly. Yet any model neglecting this is suspect. The problem would be solved in one single computation if the thermal field within the die were known and used as one of the data. Convergence to the cyclic state would be accelerated if using not the real thermal field, but a good approximation of it; but such a guess is difficult.

In [11], another approach has been proposed, based on the low cost of purely thermal computations as compared to mechanical resolution. In a first step, a coupled thermo-mechanical computation in the part is made, choosing a homogeneous initial die temperature. The heat flux from the part to the die $\phi(t)$ is extracted from the results. Then, the following strokes are computed as a much simpler problem: thermal transfer in the die only, with $\phi(t)$ as an entry. This can be repeated until the thermal cycle is stable, i.e. the fixed point of the transformation has been obtained. If necessary, a coupled die + part computation can be 
introduced every $n^{\text {th }}$ stroke, to update $\phi(\mathrm{t})$, accounting for the current thermal field in the die. Overall, a CPU time division by a factor 12 has been claimed.

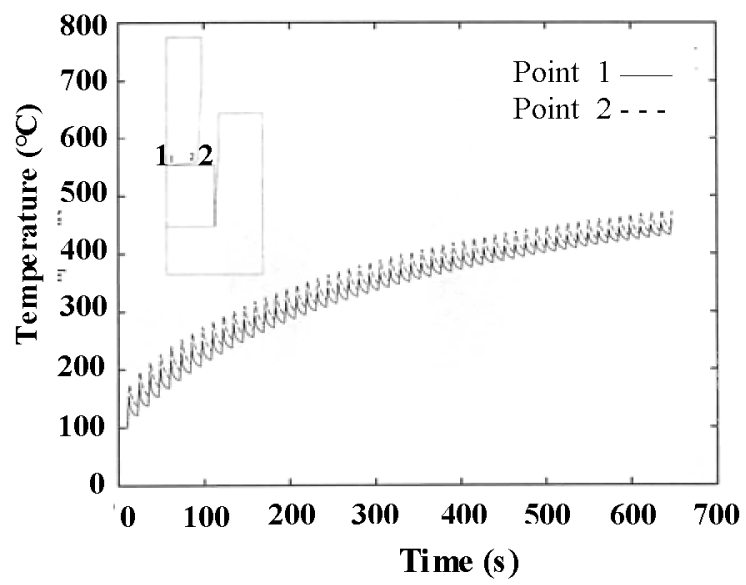

FIGURE 7. Computed surface temperature of the hot forging tools: evolution with number of pieces forged (each piece gives a temperature peak).

Note that this procedure is quite different from the one described above for pilgering: no initialization assumption is made, but the analysis of the process tells that the influence of the die temperature on the mechanical flow in the part should be small, and the time-saving comes from the decoupling of the two problems. An advantage is that the successive computations correspond to realistic, successive states of the systems, so that one of the results of the computation is how many parts must be forged before the thermal stabilized cyclic state is reached, and how much the parts forged initially differ from those forged once it has been reached. In the procedure described for pilgering, the 4 or 5 strokes computed are just successive corrections to an arbitrary initial state, they do not correspond to any physical reality.

In fact, this thermal problem is not strictly cyclic, as shown in fig.7, due to the slow drift of the temperature with part number. This is the fundamental reason of the different treatments.

\section{CONCLUSION}

Periodic, semi-continuous processes require highly intensive computations if all the strain steps are to be computed using to a purely Lagrangian formulation. If their cyclic nature is recognized, convergence acceleration to the stabilized cycle can be achieved, allowing to compute far less steps (here 4 or 5 as compared to $70 \ldots$ or 2500 ). The proposed technique is based on a careful initialization of the geometry and strain field, which has been refined using accurate experimental measurements. Of course this method, which introduces some degree of Eulerian point of view, is inadequate if end effects are to be computed.

It consists in fact in accelerating convergence by a more precise approximation of the "steady-state" (or rather cyclic state here), i.e. the fixed point of the transformation made in one deformation step. This is not so different from improving the free surface shape guess in a steady-state formulation of rolling processes. The last example on hot forging however shows that (quasi)cyclic problems in forming may be of widely different natures, and require problemspecific solutions based on a careful analysis of the characteristics of the problem under study.

\section{ACKNOWLEDGEMENT}

The work described in this paper has been financially and scientifically supported for a number of years by Cezus company, a subsidiary of AREVA Group.

\section{REFERENCES}

1. P. Standring, "Simulating Rotary Forging" in Proc. (CDROM) Workshop on Modeling of Bulk Metal Forming Processes, 17-18 May 2006, Darmstadt, Germany.

2. P. Montmitonnet, R. Logé, M. Hamery, Y. Chastel, J.-L. Doudoux, J.-L. Aubin, J. Mater. Process. Technol. 125126, 814-820 (2002).

3. B. Lodej, K. Niang, P. Montmitonnet, J.-L. Aubin, J. Mater. Process. Technol. 177, 188-191 (2006).

4. G.J. Li, S. Kobayashi, ASME J. Engng Ind. 104, 55-64 (1982)

5. K. Mori, K. Osakada, Int. J. Mech. Sci. 26, 9-10, $515-$ 525 (1984)

6. Y.S Lee, P.R. Dawson, T.B. Dewhurst, "Bulge prediction in steady state bar rolling process", Proc. NUMIFORM 89 (Fort Collins, USA, 1989) E.G. Thompson et al., Eds. A.A. Balkema, Rotterdam, 1989.

7. J.-L. Chenot, P. Montmitonnet, A. Bern, C. BertrandCorsini, Comp. Meth. Appl. Mech. Eng. 92, 2, 245-260 (1991)

8. A. Hacquin, P. Montmitonnet, J.-Ph. Guillerault, J. Mater. Process. Technol. 60, 1-4, 109-116 (1996)

9. J.-L. Aubin, E. Girard, P. Montmitonnet, "Modelling of damage in cold pilgering", in Proc. $10^{\text {th }}$ A.S.T.M. Int. Symp. on Zirconium (Baltimore, June 1993), A.M. Garde \& E.M. Bradley, eds, ASTM, STP 1245 (Philadelphia, 1995)

10. R.D Krieg, D.G. Krieg, ASME J. Pressure Vessels Technol. 99, 510-515 (1977)

11. M. P. Miles, L. Fourment, J.-L. Chenot, J. Mater. Process. Technol. 45, 643-648 (1994) 